\title{
Propriedades psicométricas da versão brasileira do Exame Geronto Psicomotor
}

\author{
Psychometric properties of the Brazilian version of the Geronto Psychomotor Exam \\ Propiedades psicométricas de la versión brasileña del Examen de Psicomotricidad de Geronto
}

Recebido: 27/09/2021 | Revisado: 04/10/2021 | Aceito: 10/10/2021 | Publicado: 12/10/2021

\author{
Feng Yu Hua \\ ORCID: https://orcid.org/0000-0003-1380-4350 \\ Universidade Federal do ABC, Brasil \\ E-mail: fengbr2@gmail.com \\ Francine Náthalie Ferraresi Rodrigues Queluz \\ ORCID: https://orcid.org/0000-0002-8869-6879 \\ Centro Universitário Adventista de São Paulo, Brasil \\ E-mail: francine.queluz@gmail.com \\ Elaine Rabelo Neiva \\ ORCID: https://orcid.org/0000-0003-0503-3234 \\ Universidade de Brasília, Brasil \\ E-mail: elaine.neiva@gmail.com \\ Marisete Peralta Safons \\ ORCID: https://orcid.org/0000-0001-9822-603X \\ Universidade de Brasília, Brasil \\ E-mail: mari7ps@gmail.com
}

\begin{abstract}
Resumo
O presente estudo verificou as evidências de validade e confiabilidade da versão brasileira do Exame Geronto Psicomotor (EGP-Br). 215 idosos, com idade média de 70,22 anos (DP: + 6,59) responderam a um questionário demográfico e foram avaliados com o EGP-Br. Os dados foram analisados por Análise Fatorial Exploratória e o alfa de Cronbach, considerando os índices de ajuste: GFI (Goodness of Fit Index), AGFI (Ajusted Goodness of Fit Index) e RMSR (Root Mean Square Residual) e a Variância Média Extraída. A estrutura fatorial apresentou três fatores: Fator 1 = Cognição $(\alpha=0,90)$; Fator $2=$ Mobilidade Articular $(\alpha=0,80)$; Fator $3=$ Equilíbrio $(\alpha=0,70)$, com bons índices de ajuste $(\mathrm{GFI}=0,99$; AGIF =0,99; RMSR =0,03). Os parâmetros psicométricos dos fatores 1,2 e 3 foram respectivamente: Validade Convergente $=0,55 ; 0,70 ; 0,50$. Validade Discriminante $=0,74 ; 0,84 ; 0,70$. Conclui-se que o EGP-Br apresenta boas evidências de validade e confiabilidade, adequadas para utilização no contexto brasileiro. Palavras-chave: Psicometria; Envelhecimento; Equilíbrio postural; Memória; Cognição.
\end{abstract}

\begin{abstract}
The present study verified the evidence of validity and reliability of the Brazilian Geronto Psychomotor Exam (EGPBr). 215 participants, mean age 70.22 years $(\mathrm{SD}:+6.59)$ answered a sociodemographic questionnaire and were evaluated using the EGP-Br. The data were analyzed using Exploratory Factor Analysis, Cronbach's alpha, considering the adjustment indices: GFI (Goodness of Fit Index), AGFI (Adjusted Goodness of Fit Index) and RMSR (Root Mean Square Residual) and the Average Variance extracted. Factor Analysis indicated a structure with three factors: Factor $1=$ Cognition $(\alpha=0.90)$; Factor $2=$ Joint Mobility $(\alpha=0.80)$; Factor $3=$ Equilibrium $(\alpha=0.70)$, with good adjustment rates $(\mathrm{GFI}=0.99 ; \mathrm{AGIF}=0.99 ; \mathrm{RMSR}=0.03)$. The psychometric parameters presented by factors 1,2 and 3 were respectively: Convergent Validity $=0.55 ; 0.70 ; 0.50$. Discriminant Validity $=0.74 ; 0.84 ; 0.70$. In conclusion, the EGP$\mathrm{Br}$ presents good evidence of validity and reliability, suitable for use in the Brazilian context.
\end{abstract}

Keywords: Psychometry; Aging; Postural balance; Memory; Cognition.

\section{Resumen}

El presente estudio verificó la evidencia de validez y confiabilidad del Examen Psicomotor de Geronto brasileño. 215 ancianos, edad media 70,22 años (DE: $+6,59)$ respondieron un cuestionario sociodemográfico y evaluados com el EGP-Br. Los datos se analizaron con el Análisis Factorial Exploratorio, alfa de Cronbach, considerando los índices de ajuste: GFI (Goodness of Fit Index), AGFI (Adjusted Goodness of Fit Index) y RMSR (Root Mean Square Residual) y la varianza promedio extraída. El Análisis Factorial indicó una estructura con tres factores: Factor $1=$ Cognición $(\alpha$ $=0,90)$; Factor $2=$ movilidad articular $(\alpha=0,80)$; Factor $3=$ Equilibrio $(\alpha=0,70)$, con buenas tasas de ajuste (GFI = 0,99; $\mathrm{AGIF}=0,99 ; \mathrm{RMSR}=0,03$ ). Los parámetros psicométricos presentados por los factores 1,2 y 3 fueron respectivamente: Validez Convergente $=0.55 ; 0,70 ; 0,50$. Validez discriminante $=0,74 ; 0,84 ; 0,70$. En conclusión, el EGP-Br presenta buena evidencia de validez y confiabilidad, adecuado para su uso en el contexto brasileño.

Palabras clave: Psicometría; Envejecimiento; Equilibrio postural; Memoria; Cognición. 


\section{Introdução}

Em 2039, o número de idosos no Brasil será maior do que o número de crianças e adolescentes, quando um a cada quatro brasileiros terá 60 anos ou mais (IBGE, 2019). Ao se considerar este acentuado envelhecimento populacional, fica claro a necessidade de desenvolver estratégias públicas para a promoção da saúde, para que a longevidade seja vivida com qualidade, mantendo a autonomia e independência dos idosos (WHO, 2016).

A autonomia diz respeito à condução e gestão da própria vida. Envolve as funções cognitivas, como. Por exemplo, o planejamento e organização das questões do cotidiano: administração financeira, organização do funcionamento doméstico, planejamento de viagens e rotinas de cuidados com a saúde. De forma complementar, a independência está relacionada também à capacidade de execução das tarefas do cotidiano, exigindo também habilidades motoras. Como em situações em que o idoso precisa se alimentar, cuidar de sua higiene e se locomover sem necessitar de auxílio de terceiros (WHO, 2012, 2015).

Percebe-se então, que, para garantir a qualidade de vida ao longo do envelhecimento, é fundamental um adequado acompanhamento das funções que estão relacionadas com os aspectos cognitivos e motores. Para este fim, existem diversos instrumentos para avaliação das capacidades motoras e cognitivas de idosos (Camara et al., 2008; César et al., 2015; Martins et al., 2017), porém são predominantemente cognitivos ou motores, sem permitir uma visão global do indivíduo ou apresentar características multidisciplinares.

Com o intuito de se obter uma visão geral da funcionalidade do idoso, abordando tanto aspectos cognitivos como motores, o Examen Géronto Psychomoteur (EGP) foi desenvolvido na França e propõe avaliar a capacidade funcional e os mecanismos compensatórios da estrutura cognitivo-motora no envelhecimento. Além disto, também auxilia na compreensão mais ampla e abrangente do indivíduo idoso, utilizando atividades ecológicas, que são necessárias no dia a dia, simulando situações cotidianas (Michel et al., 2011).

O uso do EGP também atende as duas principais queixas no envelhecimento: prejuízos na memória e no equilíbrio, além de legitimar as ações da prática profissional. Ademais, pode evitar intervenções desnecessárias, ao verificar o desempenho de funções necessárias para as atividades cotidianas e assim direcionar o projeto terapêutico para manutenção, adaptação ou reabilitação das habilidades avaliadas: coordenação motora estática e dinâmica, mobilidade passiva e ativa de membros superiores e inferiores, praxia, atenção, memória, linguagem, comunicação e noções corporais, espaciais e temporais (Michel et al., 2010).

No que diz respeito ao uso do instrumento em outros países, o EGP foi traduzido e adaptado para o uso em Portugal (Morais et al., 2016a), no Uruguai (Morais et al., 2016b), no Líbano (Faddoul et al., 2017), e no Brasil (Hua et al., 2019a), sendo que nos três primeiros países, os estudos apresentaram evidências de validade satisfatórias para as respectivas culturas. Em Portugal, as investigações vêm se ampliando, e os estudos que utilizam o EGP têm contribuído para melhor compreensão de indivíduos institucionalizados (Morais et al., 2015), com diagnósticos de Doença de Alzheimer (Morais et al., 2019) e Doença de Parkinson (Cortiçada et al., 2018).

No Brasil, o EGP foi traduzido e adaptado seguindo as etapas internacionalmente estabelecidas: 1. tradução, 2. comitê de especialistas e 3. pré-teste. As evidências de validade apresentaram índices que classificaram as equivalências semânticas, conceituais, culturais e clareza dos itens, como quase perfeitas, apontadas por coeficientes de índices de validade de conteúdo (IVC) entre 0,86 e 1,00. O coeficiente de concordância entre a avaliação dos juízes do comitê de especialistas demonstrou forte concordância, com valores de Kappa entre 0,85 e 0,96. No pré-teste, o tempo médio de duração da avaliação com o EGP foi de $52,36 \pm 7,48$ minutos e a média dos escores dos participantes foi de 91,35 \pm 9,85 (Hua et al., 2019a).

A partir desses resultados, e considerando um número cada vez maior de idosos na população mundial, percebe-se a importância de instrumentos voltados à população brasileira e que possam avaliar, simultaneamente, aspectos cognitivos e motores dos idosos, uma vez que estes poderão auxiliar em diagnósticos e subsidiar futuras intervenções. Assim, tornar um 
instrumento apto para uso requer que se obtenha vários tipos de evidências de validade, pois visa garantir que a utilização de forma confiável da versão brasileira do EGP seja adequada.

Sendo assim, o objetivo do presente estudo é verificar evidências de validade e confiabilidade da versão brasileira do Exame Geronto Psicomotor (EGP-Br).

\section{Metodologia}

\section{Delineamento do estudo}

Este trabalho consiste em um estudo metodológico de validação de um instrumento de medida para investigar as evidências de validade interna e a confiabilidade. Para este fim, foram seguidas as etapas internacionalmente estabelecidas (Mokkink et al., 2010). A pesquisa tem caráter quantitativo e adotou um desenho transversal, utilizando dados de uma amostra não probabilística.

\section{Participantes}

A amostra do estudo foi constituída por 215 voluntários, 84,7\% do sexo feminino, média de idade de 70,22 $( \pm 6,59)$ anos, escolaridade relatada de 9,56 $( \pm$ 7,23) anos de ensino formal, e 8,1\% apresentavam diagnóstico de transtorno neurocognitivo. $\mathrm{O}$ recrutamento foi por conveniência, com idosos da comunidade, programas de atenção ao idoso em instituições públicas e privadas, centros de convivência de idosos, instituições religiosas e clubes recreativos do Distrito Federal. Os critérios de inclusão foram ter 60 anos ou mais e disponibilidade de aproximadamente 1 hora para avaliação. $O$ único critério de exclusão foi apresentar restrição ao leito ou à cadeira de rodas. Todos os participantes do estudo responderam um questionário com perguntas sobre suas características pessoais, diagnóstico médico, exercício físico, dor, queda e medo de cair.

O tamanho da amostra foi adequado para as análises realizadas, considerando que o desejável é um número de 5 a 10 respondentes por item do instrumento, e que não seja menor que 100. Também foi observada a comunalidade, que é a variância comum entre os itens. Quanto menor este índice, maior a amostra necessária para uma análise robusta (Hair Jr et al., 2009; Marôco, 2010; Tabachnick \& Fidell, 2013).

\section{Instrumento}

O EGP é um teste que avalia funções motoras e cognitivas, que são habilidades fundamentais para a manutenção da saúde no envelhecimento. A versão do EGP traduzida para o português do Brasil (Hua et al., 2019a), assim como a original (Michel et al., 2011), é constituída de 17 itens: coordenação estática, equilíbrio estático, marcha, equilíbrio dinâmico, mobilidade passiva de membros superiores, mobilidade passiva de membros inferiores, coordenação motora fina de membros superiores, coordenação motora fina de membros inferiores, praxia, noção corporal, atenção, memória perceptiva, noção espacial, memória verbal, percepção, noção temporal e comunicação. Cada item pode contabilizar uma pontuação de 0 a 6 , sendo assim, o escore total do EGP tem um valor mínimo de zero e máximo de 102, e a interpretação dos escores segue a orientação de que quanto maior o valor obtido, melhor o desempenho apresentado.

\section{Procedimentos}

Inicialmente entrou-se em contato com as instituições para a autorização da coleta de dados. Em seguida, o projeto foi submetido ao comitê de ética. Após o aceite, os idosos foram convidados a participar deste estudo por meio das instituições parceiras e respeitando-se os critérios de inclusão e exclusão. 
A análise das propriedades psicométricas de um instrumento envolve aferir se o mesmo avalia o que se propõe a avaliar (Pasquali, 2017). Neste estudo, a coleta de dados para verificar as evidências de validade EGP-Br foi realizada por uma equipe formada pela pesquisadora responsável e cinco alunos de graduação do curso de Educação Física que participaram do treinamento para uso do EGP, uniformizando a aplicação e a correção dos itens.

As coletas de dados foram realizadas em abordagens individuais com duração aproximada de uma hora. Os locais estabelecidos foram organizados para melhor acomodação e privacidade. Conforme a conveniência do participante, as avaliações ocorreram nas próprias instituições que os voluntários frequentavam ou nos seus domicílios. Primeiramente os idosos assinavam ao Termo de Consentimento Livre e Esclarecido (TCLE), esclarecendo possíveis dúvidas e em seguida, respondiam ao questionário sociodemográfico e avaliados com o uso do EGP-Br.

\section{Análise dos dados}

Para verificar a estrutura fatorial do EGP foi realizada uma Análise Fatorial Exploratória (AFE), que verifica as subescalas que formam a escala total, baseada na correlação dos itens (Hair Jr et al., 2009). Esta escolha foi realizada por se tratar de uma escala multidisciplinar, em que a versão original não apresentou agrupamento dos itens. Dessa forma, não se buscou confirmar uma teoria e sim explorar qual seria a estrutura fatorial em uma amostra brasileira, verificando quais itens estariam mais correlacionados e assim, no caso de confirmar a multidimensionalidade do EGP, criar os subgrupos dos itens e nomear as variáveis latentes.

A adequação dos dados para a Análise Fatorial Exploratória foi confirmada com a estatística Kaiser-Meyer-Olkin (KMO), calculada pela somatória dos quadrados das cargas fatoriais. O coeficiente pode variar de 0 a 1 , é desejável que o valor seja > 0,80 (Marôco, 2010). O teste de Bartlet verificou a esfericidade dos dados, que é confirmada quando o valor de pé menor ou igual a 0,05. Também foi utilizada a Medida de Adequação da Amostra (MSA, measure sample adequation), que é avaliada considerando os parâmetros: > 0,80 como admirável; > 0,70 como mediano; > 0,60 como medíocre; >0,50 como ruim e < 0,50 como inaceitável (Hair Jr et al., 2009).

O método de extração foi o de componentes principais que utiliza a variância total dos itens, com rotação oblíqua para minimizar o carregamento cruzado (Tabachnick \& Fidell, 2013). A análise dos componentes principais foi utilizada porque não há a inferência de um modelo prévio do construto latente da escala. A carga fatorial mínima adotada foi de 0,40 para que o item fosse incluído no fator, pois este é o valor considerado necessário para a significância, ao considerar o tamanho da amostra do estudo que foi de 215 indivíduos (Hair Jr et al., 2009).

A retenção de fatores foi estabelecida por três critérios: I) O critério da raiz latente ou critério de Kaiser - define a retenção de fatores com Eingevalue ou autovalor maior que 1; II) O critério da variância explicada do fator - fatores que apresentaram individualmente uma variância maior que 5\% da variância total explicada; III) O critério da análise paralela realizada no software Statistical Package for the Social Sciences (SPSS) com a sintaxe apresentada na Figura 1, que retém os fatores que apresentaram Eingevalue real maior que o Eingevalue obtidos na análise realizada em um banco de dados aleatórios gerados pelo programa (Damásio, 2012; Pasquali, 2012; Saccenti \& Timmerman, 2017). 
Figura 1. Sintaxe para Análise Paralela no Statistical Package for the Social Sciences (SPSS).

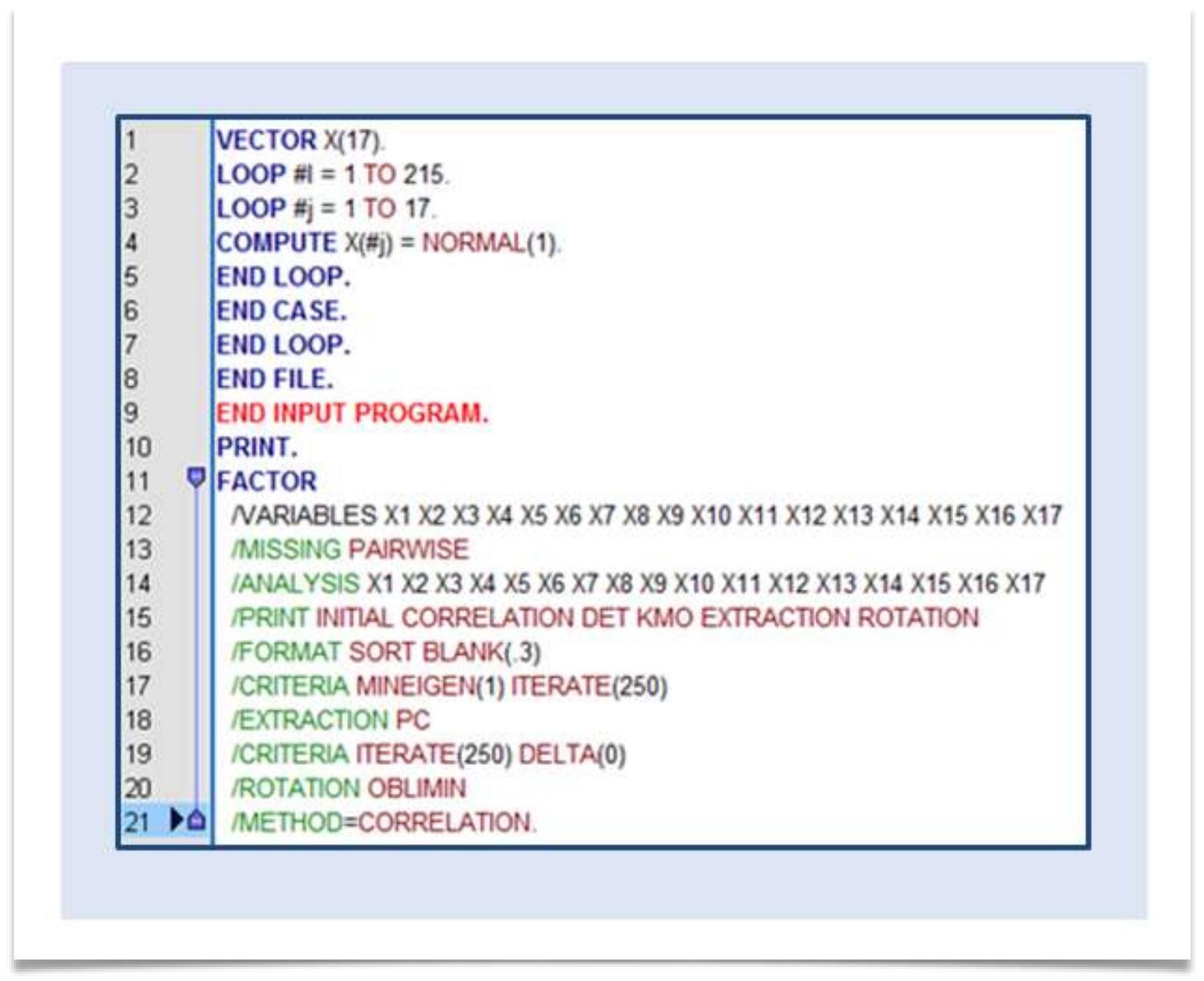

Fonte: SPSS. Elaborado pelas autoras.

O ajuste do modelo foi avaliado analisando a matriz de resíduos, e calculando os índices de ajuste GFI (Goodness of Fit Index), AGFI (Ajusted Goodness of Fit Index) e RMSR (Root Mean Square Residual); se houver um percentual maior que 50\% de resíduos não redundantes inferiores a 0,05 , entende-se que o modelo fatorial apresentado é semelhante ao modelo original, demonstrando uma boa qualidade do modelo e indicando o quanto a estrutura fatorial se ajusta aos dados (Marôco, 2010). Para verificar a validade fatorial convergente do EGP-Br utilizou-se o valor de variância média extraída (VME), valores iguais ou maiores que 0,50 são considerados satisfatórios. A raiz quadrada da VME determina o coeficiente de validade fatorial discriminante, para considerá-la adequada: o valor obtido em cada um dos fatores deve ser maior que o quadrado de todas as correlações de cada par de fatores, e os coeficientes de todas as correlações devem ser menores que 0,85 (Campbell \& Fiske, 1959; Fornell \& Larcker, 1981; Meireles \& Pasquali, 2014; Quemelo et al., 2017; Souto et al., 2016).

A confiabilidade dos fatores do EGP-Br foi verificada com o teste de consistência interna calculada pelo coeficiente de alfa de Cronbach (Cronbach, 1951), ou seja, o quanto os itens estão relacionados entre si, definindo o quão preciso é o instrumento de medida na avaliação do fenômeno que se propõe a avaliar (Hair Jr et al., 2009). Todas as análises estatísticas 
foram realizadas com auxílio do software analítico Statistical Package for the Social Sciences (SPSS) e com o editor de planilhas Microsoft Office Excel.

\section{Aspectos éticos}

O presente estudo foi submetido ao Comitê de Ética da Faculdade de Saúde da Universidade de Brasília - CEP / FS UnB. CAAE: 47735015.3.0000.0030 e aprovado pelo parecer: 1.430.179. Os cuidados éticos seguiram a resolução CNS 466/2012, publicada em 13/06/2013, do Conselho Nacional de Saúde, que regulamenta as pesquisas envolvendo seres humanos e estão de acordo com os princípios contidos na Declaração de Helsinque da Associação Médica Mundial. As condições, importância e resultados esperados da pesquisa foram apresentados e explicados para todos os voluntários, e os que concordaram em participar do estudo assinaram o Termo de Consentimento Livre e Esclarecido.

\section{Resultados}

A fatorabilidade dos dados foi garantida com a medida do $\mathrm{KMO}=0,88$, superior ao valor desejável de 0,80 . O teste de Bartlet apresentou significância estatística para esfericidade dos dados, ou seja, a matriz de covariância é similar a uma matrizidentidade. Esta verificação confere se os elementos da diagonal principal têm valor igual a um, e os demais elementos da matriz são aproximadamente zero, indicando que não apresentam correlações (Pasquali, 2012). A adequação da amostra, avaliada pelo cálculo da MSA, Medida de Adequação da Amostra , mostrou-se admirável em 13 itens: 2, 3, 7, 8, 9, 10, 11, 12, 13, 14, 15, 16 e 17; mediano em 2 itens: 5 e 6; medíocre em 1 item: 4; e o item 1 apresentou uma medida de adequação inaceitável da amostra.

Após a realização da Análise Fatorial Exploratória, encontrou-se uma estrutura com três fatores para o EGP-Br. O fator um foi predominantemente cognitivo, o fator dois foi composto pelos itens de mobilidade passiva e o fator três abordava itens que envolviam o equilíbrio. Todos os três fatores cumpriram os critérios de retenção adotados: autovalor maior que um, variância extraída maior que 5\% da variância explicada e autovalor do banco de dados maior que o autovalor de um banco aleatório, obtido por meio da análise paralela.

Os fatores foram nomeados da seguinte forma: fator um $(\mathrm{F} 1)=$ cognição; fator dois $(\mathrm{F} 2)=$ mobilidade articular; e fator três $(\mathrm{F} 3)$ = equilíbrio. $\mathrm{O}$ fator um engloba os itens mais relacionados com as tarefas que envolvem as habilidades cognitivas, $\mathrm{o}$ fator dois é composto pelos dois itens que avaliam a mobilidade das articulações dos membros inferiores e superiores, e o fator três abrange os itens que verificam as aptidões e competências necessárias para marcha e equilíbrio.

Em relação aos índices de ajustes, os parâmetros obtidos são apresentados na tabela 1 e mostram que o modelo fatorial é adequado e explica satisfatoriamente os construtos avaliados. Os três fatores apresentaram bons valores de confiabilidade, com valores do coeficiente de alfa de Cronbach igual e maiores que 0,70, valores aceitáveis para indicar precisão na medida dos construtos (Hair Jr et al., 2009; Marôco, 2010; Tabachnick \& Fidell, 2013). 
Tabela 1. Estrutura fatorial e propriedades psicométricas do Exame Geronto Psicomotor.

\begin{tabular}{|c|c|c|c|c|c|}
\hline Parâmetros avaliados & F1 & $\mathbf{F} 2$ & $\mathbf{F 3}$ & Comunalidade & MSA \\
\hline EGP 1 - Coordenação estática & & & 0,88 & 0,771 & 0,46 \\
\hline EGP 2 - Equilíbrio estático & & & 0,70 & 0,584 & 0,86 \\
\hline EGP 3 - Marcha & & & 0,63 & 0,489 & 0,84 \\
\hline EGP 4 - Equilíbrio dinâmico & & & 0,81 & 0,715 & 0,63 \\
\hline EGP 5 - Mobilidade MMSS & & 0,86 & & 0,790 & 0,72 \\
\hline EGP 6 - Mobilidade MMII & & 0,83 & & 0,754 & 0,77 \\
\hline EGP 7 - Coordenação fina MMSS & 0,77 & & & 0,651 & 0,93 \\
\hline EGP 8 - Coordenação fina MMII & & & 0,40 & 0,507 & 0,85 \\
\hline EGP 9 - Praxia & 0,78 & & & 0,628 & 0,94 \\
\hline EGP 10 - Noção corporal & 0,62 & & & 0,405 & 0,92 \\
\hline EGP 11 - Atenção & 0,71 & & & 0,611 & 0,93 \\
\hline EGP 12 - Memória perceptiva & 0,67 & & & 0,516 & 0,91 \\
\hline EGP 13 - Noção espacial & 0,84 & & & 0,796 & 0,88 \\
\hline EGP 14 - Memória verbal & 0,58 & & & 0,580 & 0,83 \\
\hline EGP 15 - Percepção & 0,81 & & & 0,666 & 0,91 \\
\hline EGP 16 - Noção temporal & 0,85 & & & 0,727 & 0,92 \\
\hline EGP 17 - Comunicação & 0,70 & & & 0,527 & 0,92 \\
\hline Eigenvalue & 6,52 & 1,75 & 1,44 & & \\
\hline Eigenvalue análise paralela & 1,60 & 1,46 & 1,27 & & \\
\hline Variância total explicada $(\%)=(57,1)$ & 38,30 & 10,30 & 8,50 & & \\
\hline Alfa de Cronbach & 0,90 & 0,80 & 0,70 & & \\
\hline Validade convergente & 0,55 & 0,70 & 0,50 & & \\
\hline Validade discriminante & 0,74 & 0,84 & 0,70 & & \\
\hline Correlação entre fatores & $\begin{array}{l}0,32 \\
(\mathrm{~F} 2)\end{array}$ & $\begin{array}{l}0,30 \\
(\mathrm{~F} 3)\end{array}$ & $\begin{array}{l}0,68 \\
(\mathrm{~F} 1)\end{array}$ & & \\
\hline \multicolumn{6}{|l|}{ Fatorabilidade: } \\
\hline \multicolumn{6}{|l|}{$\mathrm{KMO}=0,88$} \\
\hline \multicolumn{6}{|c|}{ Teste de Bartlet $=1702,20(136) ; \mathrm{p}<0,001$} \\
\hline \multicolumn{6}{|l|}{$\mathrm{GIF}=0,996$} \\
\hline \multicolumn{6}{|l|}{$\mathrm{AGIF}=0,993$} \\
\hline \multicolumn{6}{|l|}{$\mathrm{RMSR}=0,03$} \\
\hline Correlação residual $<0,05=53 \%$ & & & & & \\
\hline
\end{tabular}




\section{Discussão}

O processo de avaliação dos indícios de validade do EGP-Br foi realizado por meio de uma AFE e o modelo que emergiu desta análise apresentou evidências de validade e parâmetros psicométricos satisfatórios para um modelo com três fatores: $\mathrm{F} 1=$ Cognição, F2 = Mobilidade Articular e F3 = Equilíbrio. A escolha da AFE foi determinada por se tratar de uma estatística que explora as relações entre os construtos que compõem o instrumento para definir o modelo que melhor se ajusta aos dados analisados (Marôco, 2010). Os pressupostos teóricos que apoiaram o desenvolvimento do EGP foram os da Psicomotricidade que engloba o movimento e o intelecto (Michel et al., 2011).

Neste estudo, a estrutura fatorial encontrada é compatível com a base teórica da psicomotricidade, que é composta por habilidades físicas e cognitivas. De fato, as análises preliminares de adequação da amostra demonstraram que todos os 17 itens do EGP-Br apresentam correlações e covariações satisfatórias, atendendo os critérios necessários para proceder as análises realizadas neste estudo.

Os resultados obtidos neste estudo também reforçam a importância de um instrumento de avaliação multidisciplinar das habilidades cognitivas e motoras, pois ambas integram uma esfera global do funcionamento psicomotor, mas que podem ser divididos em subescalas: cognição, mobilidade articular e equilíbrio, corroborando a proposta de analisar a funcionalidade como um componente multidisciplinar. Para a adequada ação de uma pessoa são necessárias funções cognitivas, motoras e psíquicas, pois a interação e sobreposição dessas habilidades determinam o comportamento, os atos e tomadas de decisões do indivíduo (Falbo et al., 2016; Hua et al., 2019b; Schmitt \& Sass, 2011; Stranahan \& Mattson, 2012). O que ratifica a importância de instrumentos multidisciplinares como o EGP, para subsidiar propostas de prevenções com estimulação cognitiva e motora e protocolos de reabilitação cognitiva e motora.

O EGP contempla não apenas a avaliação, mas indiretamente também pode incentivar a adesão a programas de estimulação. Observou-se na etapa de coleta de dados, o interesse e motivação dos participantes na execução das tarefas. Frequentemente solicitavam a continuidade, dizendo-se instigados em tentar resolver as questões propostas. A ativação de novos processamentos promove o desafio e favorece a estimulação (Oliveira Jr \& Pasqualotti, 2021). O mesmo fenômeno foi observado nas tarefas predominantemente motoras. A atividade física se faz necessária em todas as etapas da vida, mas para os idosos passa a ser indispensável para manutenção da saúde (Costa et al., 2021). Os relatos dos participantes contribuem com evidências de que o EGP contempla a avaliação e estimulação de múltiplas modalidades de funções, que são integradas na complexa circuitaria do sistema nervoso humano (Hua et al., 2019b; Prado et al., 2021; Stranahan \& Mattson, 2012).

Como relatado, a execução das tarefas que compõem o EGP exige diversas habilidades de diferentes esferas. Por esta premissa, a Análise Fatorial Exploratória foi considerada a mais indicada para a análise do primeiro banco de dados, pois é a natureza dos dados que agrupa os itens nos construtos. Por sua vez, a análise fatorial confirmatória é mais adequada quando o pesquisador acredita que pode especificar os itens que compõem os construtos com base em teorias (Hair Jr et al., 2009). Este não foi o caso no presente estudo, e apoiado pelos resultados obtidos, principalmente em relação ao item $8 \mathrm{e} o$ fator 2, pois seria razoável sugerir que a coordenação motora fina de membros superiores fizesse parte da subescala motora ou até da subscala de mobilidade, que corresponde ao fator 2 , que também poderia ser incluído na escala motora.

O modelo obtido foi possível pela adoção do método de rotação oblíqua. Esta decisão foi tomada com base no entendimento de que as variáveis não são totalmente independentes entre si, o que foi confirmado pela alta correlação dos itens, o que impossibilita a rotação ortogonal (Kline, 2011), permitindo assim um ajuste do modelo que descreve a relação das variáveis com maior apoio no desempenho apresentado. No desfecho encontrado na Análise Fatorial Exploratória, o item coordenação motora fina de membros superiores, que poderia ser teoricamente designado para o construto habilidades motoras, foi alocado no construto de habilidades cognitivas. 
Um entendimento provável seria o fato das tarefas solicitadas neste item envolverem predominantemente uma das funções cognitivas mais complexa, as funções executivas, visto que nos comandos das tarefas, apesar de a descrição da execução da tarefa ser detalhada e alguns subitens terem exemplos, por não serem movimentos realizados intencionalmente no cotidiano, cabia ao avaliando criar a estratégia necessária para controlar os movimentos para o êxito na execução da tarefa. A evocação de habilidades estratégicas exige a ativação de componentes das funções executivas.

Outro item que merece atenção foi o primeiro: coordenação estática, que consiste na solicitação de que o avaliando permaneça na posição ortostática por cinco segundos. A pontuação máxima, que corresponde ao escore 6 , foi obtida por $98,1 \%$, seu MSA ficou abaixo de 0,50, além de apresentar carga cruzada com o fator 3, mas sem cumprir o quesito para retenção neste fator. A decisão de manter o item ao final do processo de validação do instrumento foi tomada, ao considerar que seriam necessários estudos com uma população que apresentasse dificuldade de se manter na posição ortostática, e então reavaliar a relevância do item.

Os parâmetros adotados para a avaliação da qualidade dos ajustes do modelo também confirmaram as evidências de validade observadas, pois todos os índices obtidos revelam que os ajustes são muito bons. Para esta classificação, os valores de GFI e AGFI precisam ser superiores a 0,95 e o RMSR inferior a 0,05 (Farrell, 2010; Garson, 2015; Hair Jr et al., 2009; Marôco, 2010). Estes resultados são apoiados pelo estudo de validação do EGP para o português europeu, a versão de Portugal também apresentou os mesmos três fatores, com as mesmas composições de itens, com apenas uma diferença: o fator dois da versão portuguesa corresponde ao fator três da versão brasileira. Em relação à variância total explicada, a estrutura fatorial do EGP-Br explicou $57,1 \%$ da variância total da escala. A versão portuguesa explicou $48 \%$ da variância total com três fatores: domínios de prevalência cognitiva, prevalência motora e restrição física (Morais et al., 2016a).

Já a versão uruguaia (Morais et al., 2016b) apresentou quatro fatores. O fator um não incluiu o item dez, como nas versões brasileira e portuguesa, este item compôs o fator três juntamente com os itens equilíbrio estático II e equilíbrio dinâmico II. O fator dois foi composto pelos itens equilíbrio estático I e equilíbrio dinâmico I, acrescido do item oito - coordenação motora fina de membros inferiores. O fator quatro corresponde ao fator dois, denominado mobilidade na versão brasileira, e que na versão portuguesa equivale ao fator três. No EGP do Uruguai cada fator foi composto da seguinte forma: fator um = itens 7 , 9 , $11,12,13,14,15,16$ e 17; fator dois = itens $1,3,8$; fator três = itens 2 , 4 e 10; fator quatro = itens 5 e 6 . A soma da variância explicada de cada um dos fatores extraídos explicou $63 \%$ da variância total.

As evidências de validade do tipo convergente e discriminante da estrutura fatorial se mostraram adequadas, pois todas apontaram índices maiores ou iguais a 0,5, que é considerado o valor mínimo para se afirmar que há evidências de validade convergente e discriminante na estrutura fatorial encontrada na escala (Fornell \& Larcker, 1981; Souto et al., 2016), demonstrando que há evidências de que os itens representam adequadamente o fator latente. E a raiz quadrada desse índice, sendo maior que a associação entre os fatores, aponta a validade discriminante, indicando que, apesar dos fatores estarem correlacionados suficientemente para integrarem uma mesma escala, há uma discriminação entre eles que justifica a divisão em subescalas (Fornell \& Larcker, 1981; Valentini \& Damásio, 2016).

A versão libanesa avaliou a consistência interna utilizando o coeficiente de alfa de Cronbach $=0,87$; Teste-reteste $=p$ valor $=0,82$. Também apontou diferença entre idosos saudáveis e idosos com Alzheimer $F(1,122)=52.86 ; p<0.0001$ (Faddoul et al., 2017). A versão original francesa do EGP apresenta diferença $\mathrm{F}(6,485)=52.29 ; \mathrm{p}<0.0001)$ entre as idades de 90 anos ou mais com relação aos demais grupos etários. Não há diferença significativa entre os demais grupos. A consistência interna foi verificada com o alfa de Cronbach $=0,83$ (Michel et al., 2010).

A fim de analisar a confiabilidade do modelo fatorial foi determinado o coeficiente alfa de Cronbach, principalmente por ser o mais utilizado em estudos do tipo transversal para indicar consistência interna ou convergência adequada. A 
confiabilidade de construto deve ser superior ou igual a 0,7 , portanto, todos os fatores apresentaram índices adequados (Hair Jr et al., 2009; Hua et al., 2019a).

A Análise da Variância Explicada, a AVE também pode ser considerada uma medida de precisão do modelo (Valentini \& Damásio, 2016) e no presente estudo o valor obtido em cada fator demonstra precisão de medida em todos os três fatores, pois evidenciaram valores iguais ou maiores que 0,50. As versões do EGP da França, Portugal, Uruguai e Líbano também apresentaram confiabilidade e precisão semelhantes, e todas as versões dos países citados apresentaram valores satisfatórios do coeficiente alfa de Cronbach.

\section{Considerações Finais}

Conclui-se que o processo de validação do Exame Geronto Psicomotor apresentou boas evidências de validade de estrutura interna e precisão para o uso no contexto brasileiro. Um instrumento desta natureza, com propriedades psicométricas, satisfatória, possibilita uma avaliação interdisciplinar que aborda ambos os aspectos cognitivos e motores, fornecendo informações para pesquisas científicas, apoiar diagnósticos, auxiliar nos prognósticos, direcionar o planejamento de estratégias de reabilitação e treinamento, além de possibilitar a avaliação da eficácia de atividades de estimulação e tratamentos cognitivo e motor ofertados para a população idosa do Brasil.

Para estudos futuros, sugere-se nova coleta de dados para a realização de uma Análise Fatorial Confirmatória, a fim de verificar se o modelo obtido na Análise Fatorial Exploratória do presente trabalho é confirmado. Além disto, futuramente, outros estudos poderiam avaliar idosos residentes em outras regiões do Brasil e população com diagnósticos específicos, característicos do envelhecimento. Por fim, o conjunto destes trabalhos propostos podem subsidiar uma normatização ampla e abrangente.

A possibilidade de digitalização do EGP também parece ser uma proposta promissora, tendo em vista as necessidades preconizadas pelo protocolo de segurança para enfrentamento da pandemia do COVID-19, que propulsionou o uso de tecnologias no cotidiano. O EGP além de avaliar as funções cognitivas e motoras, mesmo que pontualmente e de forma não sistemática, estimula as mesmas habilidades recrutadas. No formato digital exigiria novos conhecimentos e aprendizagens, que favoreceria a funcionalidade global do idoso.

\section{Referências}

Camara, F. M., Gerez, A. G., Miranda, M. L. de J., \& Velardi, M. (2008). Capacidade funcional do idoso: Formas de avaliação e tendências. Acta Fisiátrica, 15(4), 249-256.

Campbell, D. T., \& Fiske, D. W. (1959). Convergent and discriminant validation by the multitrait-multimethod matrix. Psychological Bulletin, 56(2), 81-105.

César, C. C., Mambrini, J. V. de M., Ferreira, F. R., \& Lima-Costa, M. F. (2015). Capacidade funcional de idosos: Análise das questões de mobilidade, atividades básicas e instrumentais da vida diária via Teoria de Resposta ao Item. Cadernos de Saude Publica, 31(5), 931-945. https://doi.org/10.1590/0102-311X00093214

Cortiçada, A., Vieira, F., Martins, S., Morais, A., Melo, A. P. L. dos S. B., \& Santos, S. (2018). A comunicação no envelhecimento patológico: Análise comparativa entre gerontes com demência e parkinson. Revista iberoamericana de psicomotricidad y técnicas corporales, $43,76-96$.

Costa, A. S. da., Santos, A. C. B. dos., Souza, G. C. M. S. de., Alencar, I. de. (2021). Epidemiological profile of elderly brazilians seeking physical therapy treatment: a literature review. Research, Society and Development, 10(13), e31101321113, 2021. 10.33448/rsd-v10i13.21113.

Cronbach, L. J. (1951). Coefficient alpha and the internal structure of tests. Psychometrika, 16(3), 297-334. https://doi.org/10.1007/BF02310555

Damásio, B. F., (2012). Uso da análise fatorial exploratória em Psicologia. Avaliação Psicológica. 11(2), $231-228$.

Faddoul, S. G., Abizeid, C. M., \& Albaret. (2017). Psychomotor Aging in Lebanon: Reliability and Validity of the Geriatric Psychomotor Examination. Journal of Alzheimer's Disease \& Parkinsonism, 07(05), 372.

Falbo, S., Condello, G., Capranica, L., Forte, R., \& Pesce, C. (2016). Effects of Physical-Cognitive Dual Task Training on Executive Function and Gait Performance in Older Adults: A Randomized Controlled Trial. BioMed Research International, 2016, 5812092. https://doi.org/10.1155/2016/5812092

Farrell, A. M. (2010). Insufficient discriminant validity: A comment on Bove, Pervan, Beatty, and Shiu (2009). Journal of Business Research, 63(3), 324-327. https://doi.org/10.1016/j.jbusres.2009.05.003 
Fornell, C., \& Larcker, D. F. (1981). Structural Equation Models with Unobservable Variables and Measurement Error: Algebra and Statistics. Journal of Marketing Research, 18(3), 382-388. https://doi.org/10.2307/3150980

Garson, G. (2015). Structural equation modeling. Statistical Associates Publishing.

Hair Jr, J. F., Black, W. C., Babin, B. J., \& Anderson, R. E. (2009). Multivariate data analysis (Vol. 7). Prentice Hall.

Hua, F. Y., Fernandez, J. M. G. de A., \& Safons, M. P. (2019). Tradução e adaptação do examen géronto-psychomoteur para o português do Brasil. Fisioterapia Brasil, 20(2), 213-221. https://doi.org/10.33233/fb.v20i2.2787

Hua, F. Y., Santos-Galduroz, R. F., Bagesteiro, L. B., \& Safons, M. P. (2019). Melhora do equilíbrio de idosas hígidas após treinamento cognitivo da função executiva, atenção e memória. Estudos Interdisciplinares sobre o Envelhecimento, 24(Suplemento Congresso SIAFTI), $109-119$.

IBGE. (2019). Projeção da população do Brasil e das Unidades da Federação. https://www.ibge.gov.br/apps/populacao/projecao/index.html

Kline, R. B. (2011). Principles and practice of structural equation modeling (3a ed). The Guilford Press.

Marôco, J. (2010). Análise estatística com o PASW Statistics (ex-SPSS). ReportNumber.

Martins, N. I. M., Caldas, P. R., Cabral, E. D., Lins, C. C. dos S. A., \& Coriolano, M. das G. W. de S. (2017). Instrumentos de avaliação cognitiva utilizados nos últimos cinco anos em idosos brasileiros. Ciência \& Saúde Coletiva, 24(7), 2513-2530. https://doi.org/10.1590/1413-81232018247.20862017

Meireles, E., \& Pasquali, L. (2014). Evidências de Validade e Precisão para o Inventário Fatorial dos Valores de Consumo (IFVC). Revista Sul-Americana de Psicologia, 2(2), 164-193.

Michel, S., Soppelsa, R., \& Albaret, J. (2010). Pour une légitimité du travail en géronto-psychomotricité-Pesquisa Google. Entretiens de Psychomotricité, 9093.

Michel, S., Soppelsa, R., \& Albaret, J. M. (2011). Examen géronto psychomoteur-Manuel d'application. Hogrefe.

Mokkink, L. B., Terwee, C. B., Patrick, D. L., Alonso, J., Stratford, P. W., Knol, D. L., \& et al. (2010). The COSMIN checklist for assessing the methodological quality of studies on measurement properties of health status measurement instruments: An international Delphi study. Quality of Life Research, 19(4), 539549. https://doi.org/10.1007/s11136-010-9606-8

Morais, A., Santos, S., \& Lebre, P. (2016). Psychometric properties of the Portuguese version of the Éxamen Geronto-Psychomoteur (P-EGP). Educational Gerontology, 42(7), 516-527. https://doi.org/10.1080/03601277.2016.1165068

Morais, A., Santos, S., Melo, A. P. L. dos S. B., Tuzzo, R., \& Demarchi, J. M. (2016). Propriedades psicométricas do éxamen geronto-psychomoteur (EGP): A validação em Portugal e no Uruguai. Revista iberoamericana de psicomotricidad y técnicas corporales, 41, 5-22.

Morais, A., Santos, S., \& Lebre, P. (2019). Psychomotor, Functional, and Cognitive Profiles in Older People with and without Dementia: What Connections? Dementia (London), 18(4), 1538-1553. https://doi.org/10.1177/1471301217719624

Morais, A., Santos, S., Lebre, P., Antunes, A., Varandas, P., Carneiro, P., \& et al. (2015). Perfil psicomotor de los pacientes ancianos atendidos por los centros de las Hermanas Hospitalarias (CHH) en Portugal. Informaciones psiquiátricas, 221, 57-71

Oliveira Junior, E. R. De, Pasqualotti, A. (2021). Computational thinking and cognitive processes with elderly people: systematic review. Research, Society and Development, 10(11), e563101120020. 10.33448/rsd-v10i11.20020. Disponível em: https://rsdjournal.org/index.php/rsd/article/view/20020.

Pasquali, L. (2012). Análise fatorial para pesquisadores. Brasil: LabPAM.

Pasquali, L. (2017). Validade dos Testes. Examen, 1(1), 14-48.

Prado, B. P. do ., Reis, L. A. dos ., Assis , W. C. ., Brito, F. R. ., Rabelo, L. A. N. ., Guimarães, F. E. de O. ., Britto, I. T. de ., \& Reis, L. A. dos. (2021). Physical exercise as a tool for active aging: A theoretical reflection. Research, Society and Development, 10(7),. e37710716629, 2021. 10.33448/rsd-v10i7.16629. Disponível em: https://rsdjournal.org/index.php/rsd/article/view/16629.

Quemelo, P. R. V., Milani, D., Bento, V. F., Vieira, E. R., \& Zaia, J. E. (2017). Literacia em saúde: Tradução e validação de instrumento para pesquisa em promoção da saúde no Brasil. Cadernos de Saúde Pública, 33(2), 1-15. https://doi.org/10.1590/0102-311X00179715

Saccenti, E., Timmerman, M. E., (2017). Considering Horn`s parallel analysis from a random matrix theory point of view. Psychometrika. 82(1): 186-209.

Schmitt, T. A., \& Sass, D. A. (2011). Rotation criteria and hypothesis testing for exploratory factor analysis: Implications for factor pattern loadings and interfactor correlations. Educational and Psychological Measurement, 71(1), 95-113. https://doi.org/10.1177/0013164410387348

Souto, E. P., Vasconcelos, A. G. G., Chor, D., Reichenheim, M. E., \& Griep, R. H. (2016). Validade da estrutura fatorial da escala de capital social utilizada na linha de base no ELSA-Brasil. Cadernos de Saúde Pública, 32(7), 1-12. https://doi.org/10.1590/0102-311X00101515

Stranahan, A. M., \& Mattson, M. P. (2012). Recruiting Adaptive Cellular Stress Responses for Successful Brain Ageing. Nature Reviews Neuroscience, 13(3), 209-216. https://doi.org/10.1038/nrn3151

Tabachnick, B. G., \& Fidell, L. S. (2013). Using multivariate statistics (6a ed). Pearson Education.

Valentini, F., \& Damásio, B. F. (2016). Variância Média Extraída e Confiabilidade Composta: Indicadores de Precisão. Psicologia: Teoria e Pesquisa, 32(2), 1-7. https://doi.org/10.1590/0102-3772e322225

World Health Organization (WHO). (2012). Active ageing. WHO.

World Health Organization (WHO). (2015). Ageing and health. WHO.

World Health Organization (WHO). (2016). Global health observatory data repository: Life expectancy. WHO. 